\title{
Safety and Efficacy of Four Anti-Diabetic Treatment Regimens in Patients with Type 2 Diabetes during Ramadan fasting: A Comparative Study
}

\section{Saira Burney, ${ }^{1}$ Khadija Irfan Khawaja, ${ }^{2}$ Aziz Fatima, $^{3}$ Ali Atif, ${ }^{4}$ Faisal Masud (Late) ${ }^{5}$}

\begin{abstract}
Objective: To compare the anti-hyperglycaemic effectiveness and safety of different anti-diabetic treatment regimens in fasting type 2 diabetic subjects during Ramadan.

Methods: This was a prospective four-week study of 161 subjects with type 2 diabetes (HbA1c 6.5-8.5\%) during Ramadan, at the Department of Endocrinology \& Metabolism, Services Hospital, Lahore, Pakistan. Study participants were randomized into four treatment groups: lifestyle modification only (group 1: lifestyle), metformin monotherapy (group 2: Metformin), metformin and sulfonylurea (group 3: Met/SU) and metformin and DPP4 inhibitor (group 4: Met/Sita). Anthropometric and biochemical evaluation were done one week before onset of Ramadan and in the last week of Ramadan. 134 patients completed the study protocol and repeated measures ANOVA was used to compare the groupsfor change in clinical and laboratory parameters from pre-Ramadan baseline.

Results: There was a significant decrease in mean blood glucose $(\mathrm{mg} / \mathrm{dL})$ and fructosamine $(\mu \mathrm{mol} / \mathrm{L})$ levels post-Ramadan in all treatment groups, with the greatest reduction seen in Met/Sita treatment group [(fructosamine $-35.9, p<0.01)$, mean blood glucose $(-23.6, p<0.05)]$. In the safety analysis, Met/Sita group also showed improvement in several metabolic parameters including lipid profile (cholesterol $-12.4 \mathrm{mg} / \mathrm{dL}, \mathrm{p}$ $<0.05$; LDL $-8.6 \mathrm{mg} / \mathrm{dL}, \mathrm{p}<0.05$; triglycerides $-41.9 \mathrm{mg} / \mathrm{dL}, \mathrm{p}<0.05)$ and transaminases. Only one patient among the study population reported a hypoglycaemic event (Met/SU group).

Conclusion: During Ramadan fasting, combination treatment with metformin-sitagliptin in type 2 diabetic subjects resulted in the greatest improvement in glycaemic control without causing hypoglycaemia, and was associated with improvement in lipid profile and hepatic transaminases.

Key words: Metformin, sitagliptin, fasting, Ramadan, type 2 diabetes, glucose control
\end{abstract}

\section{Introduction:}

$\mathrm{I}$ slam is the second largest world religion with an estimated 1.8 billion followers. ${ }^{1}$ Ramadan fasting is obligatory for all adult Muslims; however, exemption is granted in certain situations including any form of ill-health likely to be severely affected by fasting. ${ }^{2}$ Despite this laxity, Muslims are keen to fulfill this obligation irrespective of their illness, diabetic

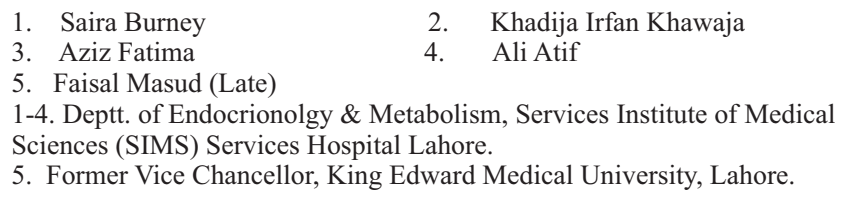

5. Former Vice Chancellor, King Edward Medical University, Lahore.

\section{Correspondence:}

Saira Burney

Deptt. of Endocrionolgy \& Metabolism, Services Institute of Medical Sciences (SIMS) Services Hospital Lahore.

$\begin{array}{ll}\text { Submission Date: } & 28-09-2020 \\ \text { 1st Revision Date: } & 23-10-2020 \\ \text { Acceptance Date: } & 8-11-2020\end{array}$

patients being no exception. An estimated 40-50 million diabetic Muslims fast during Ramadan ${ }^{3}$. As reported by the large multi-centric Epidemiology of Diabetes and Ramadan study (EPIDIAR), 79\% of type 2 diabetic subjects and $43 \%$ of type 1 observe Ramadan fasting annually. ${ }^{4}$ Many of the Muslim majority countries have the highest percentage of diabetic patients, including Pakistan, which has a reported diabetes prevalence of $\sim 12 \%$, in a population of 220million,ranking 6th among world nations ${ }^{5}$.

Ramadan is a lunar-based month and the length and timing of fasting vary each year according to the seasons and geographical location. Fasting during this month requires abstinence from food, fluids and medicines from daybreak to dusk daily ${ }^{6}$. As this is opposed to the conventional dietary pattern recommended in diabetes, fasting can be expected to 
cause erratic glycaemic control and metabolic derangements even in well-controlled diabetic patients.As the frequency of self-monitoring of blood glucose during fasting is also reduced, these glycaemic swings generally go undetected. Dehydration and stroke from prolonged fasting in summer months are also potential risks. Consequently, the first International Congress on Health and Ramadan, recommended that patients suffering from severe comorbid conditions should avoid fasting ${ }^{7}$.

Current recommendations have clearly defined categories of diabetic patients at varying degrees of risk from fasting ${ }^{8}$. The epidemiological evidence available on the impact of Ramadan fasting on diabetes control concludes that fasting is well-tolerated in stable type 2 diabetic patients treated either with diet alone or combined diet and oral hypoglycaemic agents (OHA) ${ }^{9}$. The International Diabetes Federation has issued comprehensive guidelines on diabetes management during Ramadan in collaboration with Diabetes and Ramadan International Alliance ${ }^{10}$.

For the fasting type 2 diabetic patient, the aim of treatment is to minimize the frequency of hypoglycamia and maintain euglycaemia taking into consideration religious constraints. The recent years have seen an increase in therapeutic options for glucose control in diabetes. Studies have been done on the safety and efficacy of OHAs during Ramadan targeting specific treatment modalities including sulfonylureas, metformin, biphasic insulin and DPP-IV inhibitors, however, no consensus exists about the superiority in terms of safety and efficacy of any one regimen ${ }^{11}$.

This study compared different anti-diabetic treatment regimens in terms of their anti-hyperglycaemic effectiveness and safety profile during Ramadan fasting in type 2 diabetic patients.

\section{Methods}

This was an open-label, single-centre, interventional study conducted in Department of Endocrinology \& Metabolism, Services Hospital, Lahore during the month of Ramadan. The duration of fasting on average was more than 15 hours and ambient temperatures approximately $38 \mathrm{C}$. The study was designed in accordance with Declaration of Helsinki and the protocol was reviewed and approved by the Institu- tional Review Board.

Subject recruitment was done in three phases from among the diabetic patients registered with the Department: In the first phase about three months before start of Ramadan, 252 type 2 diabetic subjects intending to fast throughout Ramadan and willing to participate in the study were enrolled and their written informed consent obtained. All subjects were reminded about the specific exemption from fasting in disease states and the potential risks of acute complications. In the second phase, screening was done for underlying complications and their glycaemic control was assessed to confirm eligibility. Final screening was carried out one month prior to Ramadan onset.

The eligibility criteria were type 2 diabetes, age more than 18 years, on either diet alone or $\leq 2$ oral anti-diabetic agents with good to moderate control (HbA1c 6.5-8.5\%), in the three months preceding Ramadan. Exclusion criteria included any contraindications either to fasting or to any of the trial medication (metformin, sufonylurea or sitagliptin), pregnancy, clinical or biochemical evidence of co-morbid conditions, advanced diabetes complications, frequent hypoglycaemic episodes, or on SGLT2 inhibitors.

One week prior to start of Ramadan, the enrolled 161 participants were called to the clinic in fasting state for baseline clinical assessment and anthropometry. Fasting capillary glucose levels were checked by point-of-care device (OptiumXceed $\AA$, Abbott Diabetes Care). Venous whole blood samples were collected for $\mathrm{CBC}$ and $\mathrm{HbA} 1 \mathrm{c}$, and serum was separated and stored at -20C for assays for liver and renal function tests, serum amylase, lipid profile and serum fructosamine.

The enrolled subjects were then assigned to 1 of 4 intervention groups based on the treatment regimen they were already taking: Group 1: lifestyle modification alone $(\mathrm{n}=37)$; Group 2: Metformin monotherapy ( $\mathrm{n}=37)$; Group 3 Metformin/ Glimepiride combination ( $\mathrm{n}=47$ ); Group 4:Metformin/ Sitagliptin combination $(n=40)$. Pre-Ramadan dose adjustment was done in accordance with standard Ramadan guidelines for achieving optimal glycaemic control.

Participants were provided with a standardized 1200 calorie diet chart and a food diary, to record the type 
and amount of food and beverages taken at pre-dawn and sunset meal throughout Ramadan. Participants were advised to shift their exercise (30 minutes walk) schedule to after the post-sunset meal. A capillary blood glucose monitoring device (Optium Xceed ${ }^{\circ}$, Abbott Diabetes Care) and adequate glucose strips were provided to each participant for glucose monitoring three times daily during Ramadan: half hour before pre-dawn meal, at mid-day and 1 hour before sunset meal with additional monitoring in case of suspected hypoglycaemia. Participants were given handouts on hypoglycaemia recognition, with instructions to break the fast in case of hypoglycaemia (BSL $<70 \mathrm{mg} / \mathrm{dl})$.

During the follow-up visit (which was done just before the end of Ramadan, to avoid a clash with the Eid festival, which immediately follows Ramadan) the food diaries, blood glucose charts and adverse event charts were collected. The total number of days fasted by each participant was also noted. Re-estimation of body weight, height and waist circumference was done and venous samples in fasting state were collected.

The primary outcome measure was change in glycaemic profile (measured by serum fructosamine and mean blood glucose (MBG) during Ramadan fasting. The secondary outcomes were change in body weight and BMI, and safety parameters (fasting lipid profile, liver and renal function)after Ramadan fasting.

\section{Statistical Analysis:}

Data were analyzed using SPSS for Windows version 11.0 (SPSS Inc., Chicago, IL, USA) and presented as means $\pm \mathrm{SD}$. Repeated measures ANOVA was done to compare the change from baseline among the different treatment groups. $\mathrm{p}<0.05$ was considered statistically significant.

\section{Results}

The sequence of study enrollment, randomization and follow-up is depicted in Figure 1. The final study population included 161 subjects out of which 134 subjects completed the four-week study per protocol with small difference in retention in each group. 27 subjects failed to complete the study with 22 being lost to follow-up and 5 compelled to interrupt fasting due to acute inter-current illness. All treatment regimens were well-tolerated by the patients. The baseline characteristics of the participants in different treatment groups were comparable, except mean body weight, which was higher in group 4 participants (Table 1).

\section{Primary outcome measures}

The changes in clinical and metabolic parameters between the four treatment groups after 4 week period of Ramadan fasting were compared by ANOVA. Glycaemic measures, including serum fructosamine ( $\mu \mathrm{mol} / \mathrm{L}$ )and mean blood glucose (MBG) levels $(\mathrm{mg} / \mathrm{dL})$ pre and post-Ramadan reflected a decline in all four treatment groups, however, Group 3 showed a significant reduction in serum fructosamine (-26.2, $\mathrm{p}<0.01)$, while in Group 4, there was a significant reduction in both serum fructosamine $(-35.9, \mathrm{p}<0.01)$ and MBG (-23.6, p<0.05) from baseline values.(Fig $2 \mathrm{a}$ and $2 \mathrm{~b})$

\section{Secondary outcome measures}

Baseline body weight $(\mathrm{kg})$ and BMI were similar between treatment groups 1-3 (mean 72.6) but higher in treatment group 4(mean 83.8). All treatment groups from 1-4 showed a significant reduction in body weight and BMI post-Ramadan: group 1 (-4.7, p $<0.01)$; group $2(-1.6, \mathrm{p}<0.05)$; group $3(-0.3, \mathrm{p}<$ $0.01)$; group $4(-0.2, \mathrm{p}<0.05)($ Fig 3$)$.

Safety parameters included tests for hepatic and renal function, serum amylase and lipid profile. At 4 weeks, there was significant reduction in serum triglycerides, cholesterol and LDL levels in treatment group 4 (cholesterol -12.4 p $<0.05$; LDL -8.6 $\mathrm{p}<0.05$; triglycerides $-41.9 p<0.05)$ but not in the other three treatment groups. Over a four week period of fasting, there was a significant improvement in transaminases in all treatment groups.(Fig 4a and 4b).

The changes in clinical and metabolic parameters between the four treatment groups after Ramadan fasting are summarized in Table 2.

No adverse events either secondary to diabetes or treatment regimens were reported in any subjects who dropped out of the study. Only one case of hypoglycaemia was reported(in group 3 (Met/SU)).

\section{Discussion}


The present study aims to add to the slowly-growing and much-needed evidence-based safe and effective management of type 2 diabetes in Ramadan by comparing the efficacy and safety of the most commonly prescribed OHAs. A majority of studies done on diabetes management during Ramadan have targeted specific treatment modalities studying the safety and efficacy of individual hypoglycaemic regimens ${ }^{14}$; studies comparing different classes of oral hypoglycaemic agents in this respect are lacking.

The primary objective of this study was to determine which if any anti-diabetic treatment group is superior in terms of improving glycaemic control and other metabolic parameters during Ramadan fasting. Keeping in view the limited time span of the study, we selected fructosamine levels and mean blood glucose among glycaemic parameters, over HbA1c, which was less likely to reflect change in glycaemic control over the period of one month. The results showed that the metformin-sitagliptin combination was superior to other treatment regimens in improving both glycaemic control and other clinical and metabolic parameters including body weight, lipid profile and hepatic transaminases. There was no significant difference between the treatment groups in the incidence of hypoglycemic episodes.

Dawn to dusk fasting daily throughout Ramadan involves alteration in diet, sleep and activity patterns; however, whether this has an impact on glycaemic and other metabolic variables is controversial. A recent meta-analysis of clinical trials on the over-all effect of Ramadan fasting in diabetic patients noted many conflicting results ${ }^{15}$. These discrepancies in study findings may be explained by variation in fasting duration, dietary norms and medication regimen between different study populations. Several studies have reported no change in serum $\mathrm{HbAlc}$ and fructosamine levels during Ramadan fasting while others have reported a decrease in both values during Ramadan $^{16}$.

In our study, the Met/Sita Group showed a significant reduction in blood glucose levels over the 4-week period of Ramadan. A number of studies have assessed the role of DPPIV inhibitors in fasting type 2 diabetic patients. VECTOR, a UK-based comparative study in type 2 diabetic patients fasting during Ramadan proved that subjects on vildagliptin- metformin combination had significantly improved HbA1c post Ramadan, better tolerability and treatment adherence and reduced incidence of hypoglycaemic episodes compared to a sulphonylureametformin treatment. Sifri et al in a large multi-centre study concluded that switching to a sitagliptin-based regimen reduced the risk of hypoglycaemia in comparison to a gliclazide-based regimen. In the VIRTUE study, vildagliptin therapy was associated with fewer hypoglycameic episodes compared to SUbased regimen in a large cohort study ${ }^{17}$.

Clinical parameters of body weight and BMI showed a significant decrease across all four groups at the end of Ramadan, which can be explained by the overall calorie reduction during Ramadan as participants across the four groups were provided with standardized calorie restricted diet chart appropriate for their BMI. Most studies have reported similar results of reduction in body weight and BMI in response to Ramadan fasting. In healthy, non-diabetic subjects, Salehi et al reported a significant decrease in body weight and BMI following complete fasting in Ramadan $^{18}$. An Algerian study by Khaled and Belbarouton 276 type 2 diabetic females fasting in Ramadan reported significant weight loss $(-3.12 \mathrm{~kg}$; $\mathrm{p}$ $<0.01)$ together with a decrease in meal frequency and energy consumption ${ }^{19}$. A study on 137 healthy Jordanian adults grouped into overweight, normal and under-weight reported significant weight reduction across all groups.

In our study, the serum levels of triglycerides, total cholesterol and LDL were similar in all participants at baseline. The metformin-sitagliptin treatment group showed a significant decrease in all lipid fractions post-Ramadan; whereas the change was not significant in any of the other three groups. This result cannot be explained by the significant reduction in body weight alone which was observed in all four treatment groups. The effect of Ramadan fasting on lipid profile has also been the topic of a number of studies with variable results depending on the nature and amount of food consumption. Some studies have correlated this change with the change in body weight due to altered dietary regimen during Ramadan, decreased activity and cultural parameters ${ }^{20}$. Similar results were reported by Adlouni et al. in Morocco with a significant reduction in total cholesterol and triglyceride levels; on the other hand, a Kuwait study 
showed no significant change in lipid parameters ${ }^{21,22}$.

An additional finding was a significant drop in hepatic transaminases, blood urea and creatinine after a four-week fasting period observed in treatment group 4 . The role of metformin in reversing aminotransferase abnormalities is established in obese insulin-resistant mice with fatty liver disease ${ }^{23}$. Human trials with metformin have shown mixed results with several pilot studies on subjects with NASH treated with metformin reporting significant reduction in mean transaminase concentrations than dietary treatment alone ${ }^{24}$. Various small studies have also demonstrated the efficacy of sitagliptin in type 2 diabetes with NAFLD in improving hepatic trans$\operatorname{aminases}^{25}$.

The EPIDIAR study reported a 5-fold and 7.5-fold increase in incidence of hyperglycaemia and hypoglycaemia respectively in fasting type 2 diabetic subjects ${ }^{4}$. In our study, only one case of hypoglycaemia occurred in group 3 (metformin/glimepiride). This finding may be explained by the dietary guidelines provided to the patient, which prescribed slow absorbing complex carbohydrates in the pre-dawn meal, and exclusion of high-risk diabetic subjects with brittle control or those prone to hypoglycaemic episodes.

Our study had certain limitations. Our treatment groups were defined by the pre-existing therapy, and this precluded blinding of either the participants or the investigators. The very low incidence of hypoglycaemia seen could have been due to failure on the part of the participants to check blood glucose at the appropriate times. We recommend that future studies may use continuous glucose monitoring to get a better picture of hypoglycaemia during a fast.

\section{Conclusion}

Type 2 diabetic subjects with moderately wellcontrolled diabetes can fast safely in Ramadan, with appropriate modification of medication regimen tailored for each patient. In our study, metforminsitagliptin combination was a safe and effective therapeutic option in type 2 diabetes in terms of effectiveness in achieving glycaemic control compared to other treatment groups. There is a need for more research on the comparative effectiveness of various therapeutic options, in fasting diabetic patients.
Figure 1: Consort Diagram:

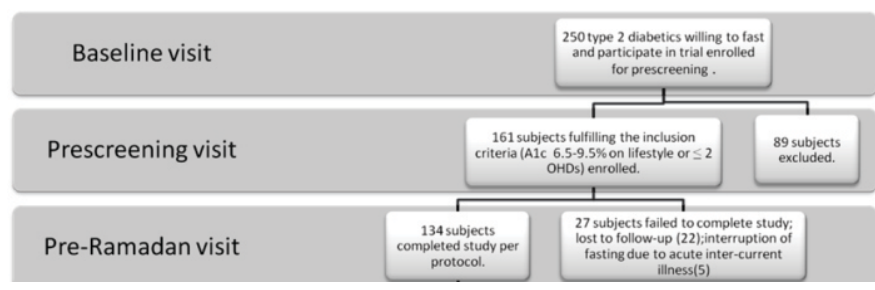

Total number of participants was 161. The groups are 1: Lifestyle modification; 2: Metformin monotherapy; 3. Metformin/Sulfonylurea combina-

Table 1: Baseline characteristics of the study population:

\begin{tabular}{|c|c|c|c|c|c|}
\hline $\begin{array}{c}\text { Baseline Characteristics } \\
\text { (Mean) }\end{array}$ & $\begin{array}{c}\text { Group } \\
1\end{array}$ & $\begin{array}{c}\text { Group } \\
2\end{array}$ & $\begin{array}{c}\text { Group } \\
3\end{array}$ & $\begin{array}{c}\text { Group } \\
4\end{array}$ & Sig. \\
\hline $\mathrm{n}$ & 37 & 37 & 47 & 40 & \\
\hline Weight & 72.1 & 72.1 & 74.3 & 83.9 & $.000 *$ \\
\hline BMI & 28.05 & 28.68 & 28.58 & 31.22 & $.011^{*}$ \\
\hline Bilirubin(mg/dl) & 0.47 & 0.48 & 0.43 & 0.50 & .631 \\
\hline $\operatorname{ALT}(\mathrm{IU} / \mathrm{L})$ & 42.00 & 39.44 & 31.80 & 42.91 & .237 \\
\hline AST (IU/L) & 30.05 & 32.16 & 26.00 & 32.47 & .266 \\
\hline Urea (mg/dl) & 27.05 & 31.32 & 29.02 & 31.71 & .385 \\
\hline Creatinine $(\mathrm{mg} / \mathrm{dl})$ & .78 & .78 & .81 & .85 & .678 \\
\hline Cholesterol(mg/dl) & 189.74 & 186.76 & 194.09 & 186.06 & .844 \\
\hline Triglyceride(mg/dl) & 164.74 & 168.12 & 203.69 & 229.79 & .251 \\
\hline HDL (mg/dl) & 39.95 & 42.08 & 40.40 & 39.74 & .858 \\
\hline $\mathrm{LDL}(\mathrm{mg} / \mathrm{dl})$ & 112.47 & 107.48 & 111.73 & 99.91 & .460 \\
\hline Amylase (U/L) & 79.13 & 67.40 & 62.98 & 67.24 & .314 \\
\hline Fructosamine $(\mu \mathrm{mol} / \mathrm{L})$ & 282.61 & 285.04 & 298.38 & 307.40 & .293 \\
\hline HbAlc(\%) & 7.01 & 7.36 & 7.18 & 7.58 & .355 \\
\hline
\end{tabular}

tion; 4: Metformin/ DPP4 combination. Values reflect change from baseline. BMI: Body mass index. MBG: mean blood glucose. ALT: Alanine aminotransferase. AST: Aspartate aminotransferase

Fig 2: Change in glycaemic parameters from baseline after Ramadan fasting in different treatment

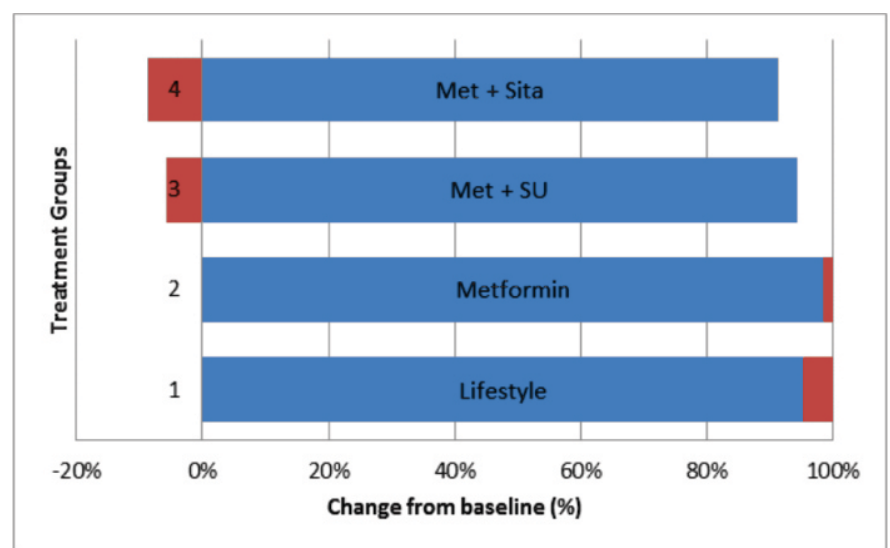

groups: The groups are 1: Lifestyle modification; 2: 
Metformin monotherapy; 3. Metformin/Sulfonylurea combination; 4: Metformin/ DPP4 combination; Blue: baseline; Red: change from baseline

\section{2a. mean blood glucose}

\section{2 b. serum fructosamine}

Fig.3: Change in weight from baseline after

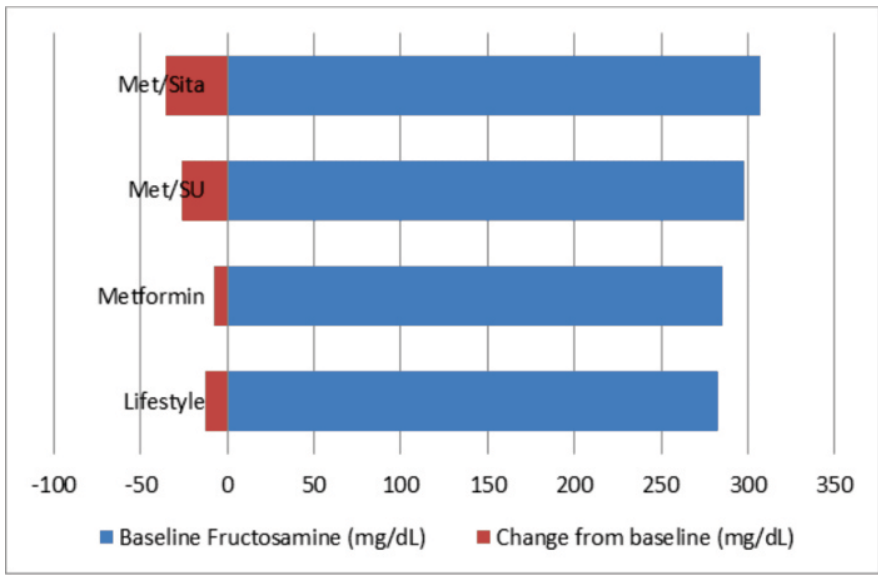

Ramadan fasting in different treatment groups: The groups are 1: Lifestyle modification; 2: Metformin monotherapy; 3. Metformin/ Sulfonylurea combination; 4: Metformin/ DPP4 combination; Blue: baseline; Red: change from baseline

Fig 4: Safety of different treatment groups during

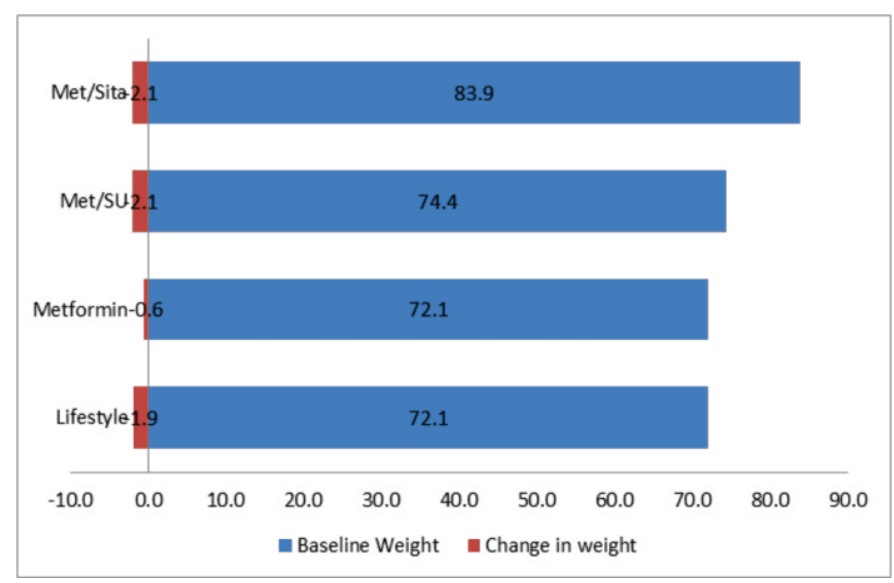

Ramadan fasting. Percentage change from baseline after Ramadan fasting in: a. metabolic parameters; b. lipid profile

The groups are 1: Lifestyle modification; 2: Metformin monotherapy; 3. Metformin/ Sulfonylurea combination; 4: Metformin/ DPP4 combination

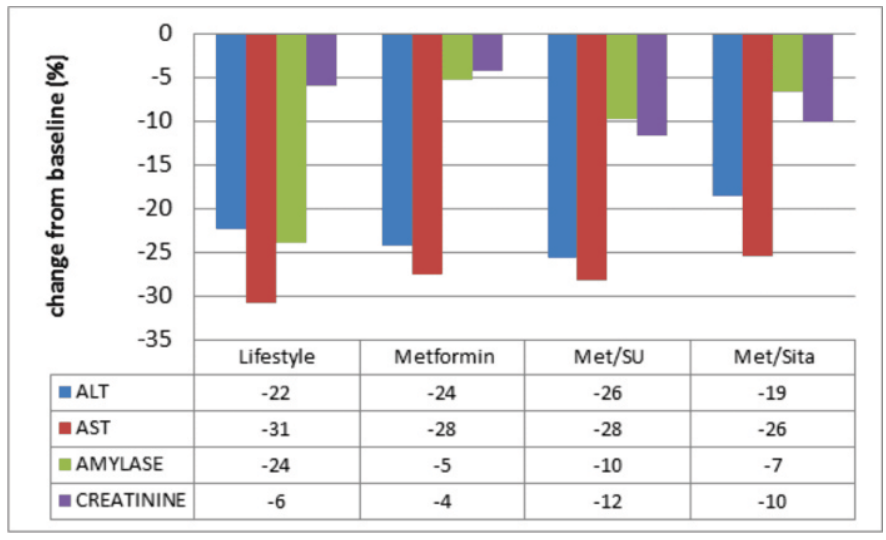

Fig 4a:

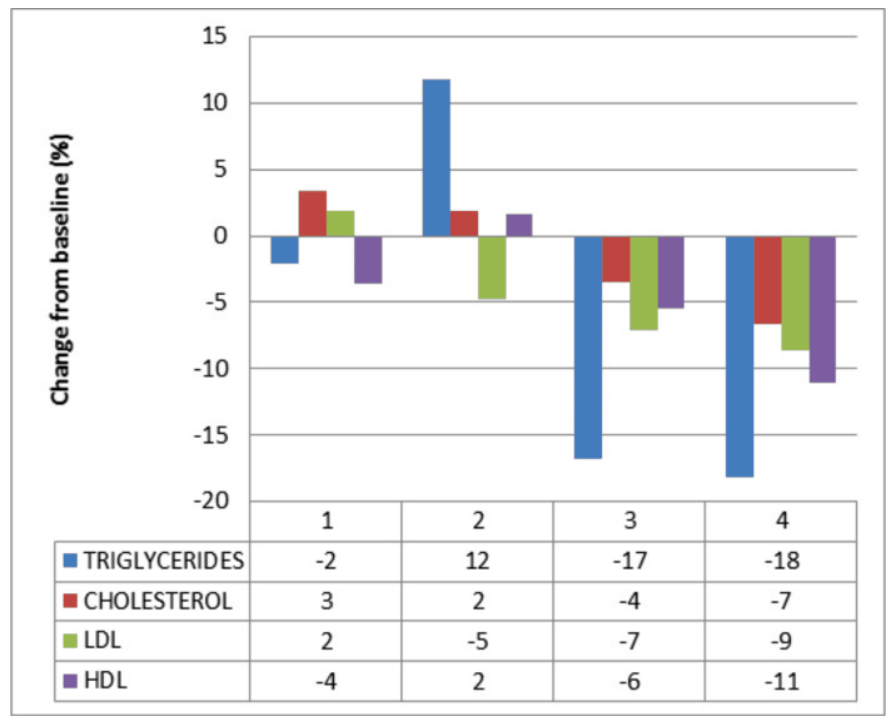

Fig 4b:

Table 2: Table 2: ANOVA showing in clinical and metabolic parameters from baseline after Ramadan fasting in different treatment groups:

\begin{tabular}{lccccc}
\hline \multicolumn{1}{c}{$\begin{array}{c}\text { Parameter } \\
\begin{array}{l}\text { (Mean change from } \\
\text { baseline) }\end{array}\end{array}$} & $\mathbf{1}$ & $\mathbf{2}$ & $\mathbf{3}$ & $\mathbf{4}$ & Sig. \\
\hline Weight $(\mathrm{Kg})$ & -1.40 & -0.40 & -1.60 & -1.77 & 0.051 \\
BMI & -0.47 & -0.16 & -0.35 & -0.18 & 0.056 \\
Bilirubin(mg/dl) & 0.07 & 0.05 & 0.08 & 0.01 & 0.670 \\
ALT (IU/L) & -9.37 & -9.67 & -8.16 & -7.97 & 0.974 \\
AST (IU/L) & -9.26 & -8.96 & -7.36 & -8.29 & 0.923 \\
Urea (mg/dl) & -2.16 & -2.63 & -1.91 & -3.70 & 0.788 \\
Creatinine (mg/dl) & -0.05 & -0.03 & -0.10 & -0.09 & 0.271 \\
Cholesterol (mg/dl) & 6.47 & 3.50 & -6.87 & -12.38 & 0.144 \\
Triglyceride (mg/dl) & -3.42 & 19.79 & -34.22 & -41.88 & 0.147 \\
HDL (mg/dl) & -1.42 & 0.71 & -2.31 & -4.41 & 0.207 \\
LDL (mg/dl) & 2.16 & -5.13 & -8.00 & -8.61 & 0.396 \\
Amylase (U/L) & -18.97 & -3.54 & -6.60 & -4.50 & 0.087 \\
Fructosamine( $\mu \mathrm{mol} / \mathrm{L})$ & -12.67 & -8.17 & -26.20 & -35.97 & $0.025^{*}$ \\
MBG(mg/dl) & +7.23 & +2.97 & -11.27 & -23.56 & $0.045^{*}$ \\
\hline
\end{tabular}

The groups are 1: Lifestyle modification; 2: 
Metformin monotherapy; 3. Metformin/Sulfonylurea combination; 4: Metformin/ DPP4 combination. Values reflect change from baseline. BMI: Body mass index. MBG: mean blood glucose. ALT: Alanine aminotransferase. AST: Aspartate aminotransferase. * indicates level of significance $p<0.05$.

\section{Author's Contribution}

SB: Concept, Design, Writing

KIK: Concept, Design, Data Analysis, Study Conduct

AF: Data Analysis, Writing, Review

AA: Trial Protocol Implementation

FM: Design, Concept, Clinical Analysis

\section{References}

1. Afifi ZE. Daily practices, study performance and health during the Ramadan fast. J R Soc Health. 1997 Aug; 117(4):231-5. doi: 10.1177/ 146642409711700406. PMID: 9375486.

2. Finch GM, Day JE, Razak, Welch DA, Rogers PJ. Appetite changes under free-living conditions during Ramadan fasting. Appetite. 1998 Oct;31(2):159-70. doi: 10.1006/appe.1998.0164. PMID: 9792730.

3. Rashed AH. The fast of Ramadan. BMJ. $1992 \mathrm{Feb}$ 29;304(6826):521-2. doi: 10.1136/ bmj.304.6826. 521. PMID: 1559053; PMCID: PMC1881417.

4. Salti I, Bénard E, Detournay B, Bianchi-Biscay M, Le Brigand C, Voinet C, Jabbar A; EPIDIAR study group. A population-based study of diabetes and its characteristics during the fasting month of Ramadan in 13 countries: results of the epidemiology of diabetes and Ramadan 1422/2001 (EPIDIAR) study. Diabetes Care. 2004 Oct;27(10):2306-11. doi: 10. 2337/ diacare.27.10.2306. PMID: 15451892.

5. Aamir AH, Ul-Haq Z, Mahar SA, Qureshi FM, Ahmad I, Jawa A et al Diabetes Prevalence Survey of Pakistan (DPS-PAK): prevalence of type 2 diabetes mellitus and prediabetes using HbA1c: a populationbased survey from Pakistan. BMJ Open. 2019 Feb 21;9(2): e025300. doi: 10.1136/bmjopen-2018025300. PMID: 30796126; PMCID: PMC6398762.

6. Recommendations for Management of Diabetes During Ramadan Monira Al-Arouj, Radhia Bouguerra, John Buse, Sherif Hafez, Mohamed Hassanein, Mahmod Ashraf Ibrahim et al Diabetes Care Sep 2005, 28 (9) 2305-2311; DOI: 10.2337/ diacare. 28.9. 2305

7. Khaled BM, Bendahmane M, Belbraouet S. Ramadan fasting induces modifications of certain serum components in obese women with type 2 diabetes. Saudi Med J. 2006 Jan; 27(1):23-6. PMID:
16432588 .

8. Ibrahim M, Davies MJ, Ahmad E, et al Recommendations for management of diabetes during Ramadan: update 2020, applying the principles of the ADA/ EASD consensus BMJ Open Diabetes Research and Care 2020; 8:e001248. doi: 10.1136/bmjdrc-2020001248

9. Khatib FA, Shafagoj YA. Metabolic alterations as a result of Ramadan fasting in non-insulin-dependent diabetes mellitus patients in relation to food intake. Saudi Med J. 2004 Dec; 25(12):1858-63. PMID: 15711655.

10. Hassanein M, Al-Arouj M, Hamdy O, Bebakar WMW, Jabbar A, Al-Madani A et al International Diabetes Federation (IDF), in collaboration with the Diabetes and Ramadan (DAR) International Alliance. Diabetes and Ramadan: Practical guidelines. Diabetes Res ClinPract. 2017 Apr;126:303316. doi: 10.1016/j.diabres.2017.03.003. Epub 2017 Mar 12. PMID: 28347497.

11. MudherMikhael E. Effectiveness and Safety of Newer Antidiabetic Medications for Ramadan Fasting Diabetic Patients. J Diabetes Res. 2016; 2016: 6962574. doi: 10.1155/2016/6962574. Epub 2016 Aug 24. PMID: 27642611; PMCID: PMC5013205.

12. Brunton S. GLP-1 receptor agonists vs. DPP-4 inhibitors for type 2 diabetes: is one approach more successful or preferable than the other? Int J ClinPract. 2014 May;68(5):557-67. doi: 10.1111/ ijcp. 12361. Epub 2014 Feb 6. PMID: 24499291; PMCID: PMC4238422.

13. Makrilakis K. The Role of DPP-4 Inhibitors in the Treatment Algorithm of Type 2 Diabetes Mellitus: When to Select, What to Expect. Int J Environ Res Public Health. 2019 Jul 30;16(15):2720. doi: 10. 3390/ ijerph16152720. PMID: 31366085; PMCID: PMC6696077.

14. Aziz KM. Fasting during Ramadan: efficacy, safety, and patient acceptability of vildagliptin in diabetic patients. Diabetes MetabSyndrObes. 2015 Apr 16;8: 207-11. doi: 10.2147/DMSO.S54683. PMID: 25931826 ; PMCID: PMC4404947.

15. Aydin N, Kul S, Karadağ G, Tabur S, Araz M. Effect of Ramadan fasting on glycaemic parameters \& body mass index in type II diabetic patients: A metaanalysis. Indian J Med Res. 2019 Dec;150(6):546556. doi: 10.4103/ijmr.IJMR_1380_17. PMID: 32048618; PMCID: PMC7038805.

16. Bener, Abdulbari\& Al-Hamaq, AbdullaO\&Öztürk, Mustafa \&Catan, Funda\&Haris et al (2018). Effect of Ramadan Fasting on Glycemic Control and other Essential Variables in Diabetic Patients. Annals of 
African Medicine.17.196.10.4103/aam.aam $63 \quad 17$

17. Klocker N, Belkhadir J, El Ghomari H, Mikou A, Nasciri M, Sabri M. Effects of extreme chronobiological diet alternations during Ramadan on metabolism in NIDDM with oral treatment. Istanbul, Turkey: Second International Congress on Health and Ramadan, FRSMR (Hassan II Foundation for Scientific and Medical Research on Ramadan) Istanbul; 1997. Dec 1-3, pp. 78-9. [Google Scholar]

18. Fernando HA, Zibellini J, Harris RA, Seimon RV, Sainsbury A. Effect of Ramadan Fasting on Weight and Body Composition in Healthy Non-Athlete Adults: A Systematic Review and Meta-Analysis. Nutrients. 2019 Feb 24;11(2):478. doi: 10.3390/ nu11020478. PMID: 30813495; PMCID: PMC6412279.

19. Chentli F, Azzoug S, Amani Mel A, Elgradechi A. Diabetes mellitus and Ramadan in Algeria. Indian J EndocrinolMetab. 2013 Oct;17(Suppl 1):S295-8. doi: 10.4103/2230-8210.119622. PMID: 24251192; PMCID: PMC3830338.

20. Nakamura H, Ito S, Ebe N, Shibata A. Renal effects of different types of protein in healthy volunteer subjects and diabetic patients. Diabetes Care. 1993 Aug;16(8):1071-5. doi: 10.2337/diacare.16.8.1071. PMID: 8375235.

21. Franz MJ, Bantle JP, Beebe CA, Brunzell JD,
Chiasson JL, Garg A et al American Diabetes Association. Nutrition principles and recommendations in diabetes. Diabetes Care. 2004 Jan;27Suppl 1:S36-46. doi: 10.2337/diacare.27.2007.s36. PMID: 14693924.

22. Institute of Medicine. 2005. Dietary Reference Intakes for Energy, Carbohydrate, Fiber, Fat, Fatty Acids, Cholesterol, Protein and Amino Acids. Washington, DC: The National Academies Press. http://doi,org/10,17226/10490.

23. Lin HZ, Yang SQ, Chuckaree C, Kuhajda F, Ronnet G, Diehl AM. Metformin reverses fatty liver disease in obese, leptin-deficient mice. Nat Med. 2000 Sep; 6(9): 998-1003. doi: 10.1038/79697. PMID: 10973319.

24. Kazemi R, Aduli M, Sotoudeh M, Malekzadeh R, Seddighi N, Sepanlou SG, Merat S. Metformin in nonalcoholic steatohepatitis: a randomized controlled trial. Middle East J Dig Dis. 2012 Jan;4(1):16-22. PMID: 24829630; PMCID: PMC4017700.

25. Iwasaki $T$, Yoneda M, Inamori M, Shirakawa J, Higurashi T, Maeda $\mathrm{S}$ et al Sitagliptin as a novel treatment agent for non-alcoholic Fatty liver disease patients with type 2 diabetes mellitus. Hepatogastroenterology. 2011 Nov-Dec;58(112):2103-5. doi: 10.5754/hge11263. PMID: 22024083. 